\title{
Modification of the LM124 Single Event Transients by Load Resistors
}

\author{
F. J. Franco, I. López-Calle, J. G. Izquierdo and J. A. Agapito
}

\begin{abstract}
The influence of a load resistor on the shape of the single event transients was investigated in the LM124 operational amplifier by means of laser tests. These experiments indicated that, as a general rule, load resistors modify the size of the transients. SPICE simulations helped to understand the reasons of this behavior and showed that the distortion is related to the necessity of providing or absorbing current from the load resistor, which forces the amplifier to modify its operation point. Finally, load effects were successfully used to explain the distortion of single event transients in typical feed-back networks and the results were used to explain experimental data reported elsewhere.
\end{abstract}

Index Terms-Laser irradiation, LM124, load effects, operational amplifier, single event transients, two-photon absorption.

\section{INTRODUCTION}

$\mathbf{O}$ PERATIONAL amplifiers (op amps) make up a set of useful active devices for electronic design. In the case of radiation-tolerant systems, many authors have investigated the action of heavy ions or pulsed lasers on the generation of single event transients (SETs) in popular operational amplifiers [1]-[11]. In particular, one of the most studied devices is the LM124 [12].

One of the main characteristics of the operational amplifiers is their versatility. An example of this is the capability of these devices to bias output loads if a critical current value, so-called short-circuit current, is not exceeded. Previous papers have dealt with the influence of the output resistance in comparators showing that there is a clear dependence of the transient shape on the resistance value [1], [13]-[15]. In fact, these works revealed that the cross-section as well as the duration of the single events strongly depend on the resistive load connected to the device output. Sharing similar internal blocks, operational amplifiers are not very different from comparators so it would be interesting to investigate the way that the single event transients are modified by the presence of a resistive load.

This work was supported in part by the EMULASER project (CDTI/PNE034/2006) and by the Spanish MCINN through Grant CTQ2008-02578/BQU, and Consolider SAUUL CSD2007-00013. Finally, I. López-Calle gratefully acknowledges the grant offered by the "Miguel Casado San José" private foundation.

F. J. Franco, I. López-Calle, and J. A. Agapito are with the Departamento de Física Aplicada III, Facultad de Físicas, Universidad Complutense de Madrid (UCM), 28040 Madrid (Spain) (e-mail: isabelcalle, monti, agapito@ fis.ucm.es).

J. G. Izquierdo is with the Servicio de Espectroscopía Multifotónica y de Femtosegundo, CAI de Espectroscopía, Facultad de Químicas, Universidad Complutense de Madrid (UCM), 28040 Madrid (Spain) (e-mail: jegonzal@quim.ucm.es).
In recent years, the influence of this parameter was also indirectly investigated by other authors, such as Boulghassoul et al. [16]. In this paper, the value of an output feedback resistor of the LM124 \& OP-27 placed on an actual satellite application was changed in order to find the worst case situation. Thus, it was discovered that the trend was that the lower the resistor value, the smaller the transients although no explication of this behavior was provided. In a previous paper [5], Sternberg et al. simulated an LM124 inside a noninverting network with a gain of 11 and changed the resistor values. Thus, SETs were simulated using several pairs of resistors ranging from $10 \mathrm{k} \Omega-1 \mathrm{k} \Omega$ to $1 \mathrm{M} \Omega-100 \mathrm{k} \Omega$ observing a strong dependence on the resistor values even though the ratio between them was the same. This astonishing result was attributed by the authors to a coupling between the resistance values, gain, and bandwidth.

However, some experiments performed at the Universidad Complutense de Madrid show that the dependence of the SETs on the feed-back network resistor values could be also related to simple load effects on the output of the operational amplifier. In fact, the authors believe that, at least in large transients involving changes of output voltage sign, the distortion of the SETs is linked to the size of the current provided or accepted by the output of the operational amplifier, which helps some of the internal transistors to recover the stable state. Besides, these results seem to be in agreement with the experiments reported by Buchner et al. in 2008 [17].

\section{EXPERIMENTAL SET-UP}

\section{A. Laser configuration}

The experiments were performed at the UCM Multiphotonic Spectroscopy and Femtosecond Facility using a Ti:Sapphire laser followed by a regenerative amplifier. The laser wavelength is tunable between 300-3000 nm. For two-photon absorption processes in silicon, 60-fs laser pulses at a frequency of $1 \mathrm{kHz}$ and a wavelength of $1300 \mathrm{~nm}$ was fixed. The energy was measured with a typical commercial powermeter and set to $1.2 \mathrm{~nJ}$.

The device was mounted on a motorized $x y z$ stage with 0.1 $\mu \mathrm{m}$ resolution and it could be observed with an infrared CCD camera to allow the correct placement of the laser. Laser beam was focused with a 50x long working-distance microscope objective, appropriate for infrared light and making the spot diameter on the order of $1.5 \mu \mathrm{m}$. Afterwards, a sweep along the $z$-axis was performed in order to store a large set of output transients and, this way, to statistically validate the results. This kind of test could be done since the laser wavelength 


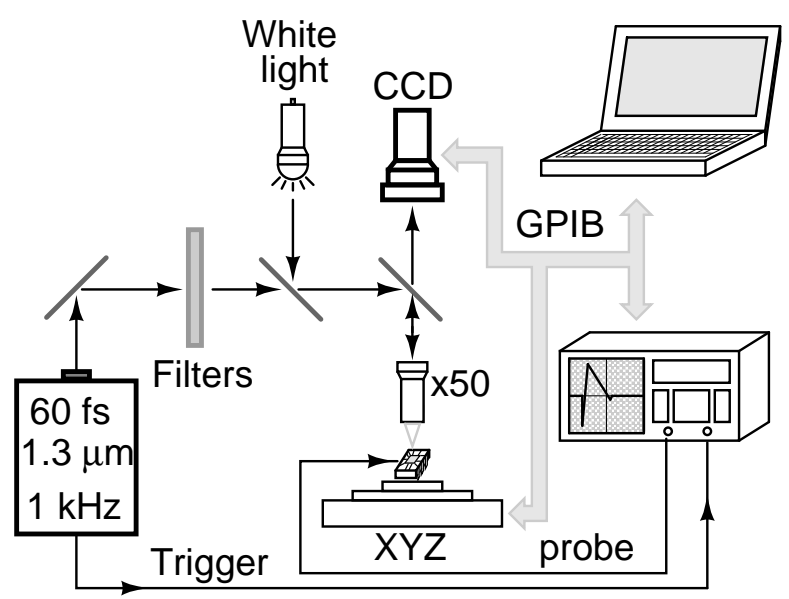

Fig. 1. Laser configuration and test set-up.

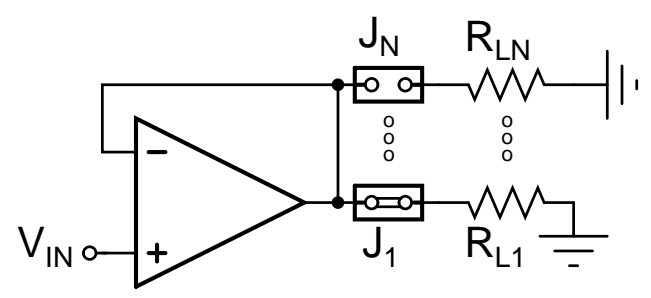

Fig. 2. Electric configuration of the LM124 operational amplifier during the laser irradiation. Jumpers were used instead of other devices such as analog switches due to the absence of parasitic capacitances. All of them were removed for unloaded configuration.

was chosen to induce two-photon absorption processes, able

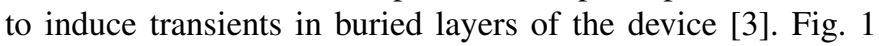
shows a graphical description of the laser system.

\section{B. Electronic set-up}

The LM124 operational amplifier, in quad CERDIP package, was mechanically decapsulated and tested as follows. The amplifier to be tested was configured as a buffer the input of which, $V_{I N}$, was connected either to ground or to an external source of $-1 \mathrm{~V}$. These values were selected in order to modify the bias point of the output stage as it will be explained later (Section $\mathbb{E - A}$ ). A set of jumpers allowed the selection of the $1 \%$-tolerance resistors with different values to load the operation amplifier output (Fig. W). Finally, the device was biased with $\pm 15 \mathrm{~V}$ power supplies. The other three operational amplifiers were configured as voltage followers and the input connected to ground.

The output was connected to a digital oscilloscope with 8$\mathrm{pF}$ probes that was triggered by means of an external signal coming from the laser (Fig. W). Data preceding the laser impact no longer than $10 \mu$ s were also saved to determine the DC output value before the event. Given that the step was $10 \mathrm{~ns}$, every set of data amounted to 10.000 points that were saved by a specific LabView application, which also controlled all the devices by means of the GPIB protocol.

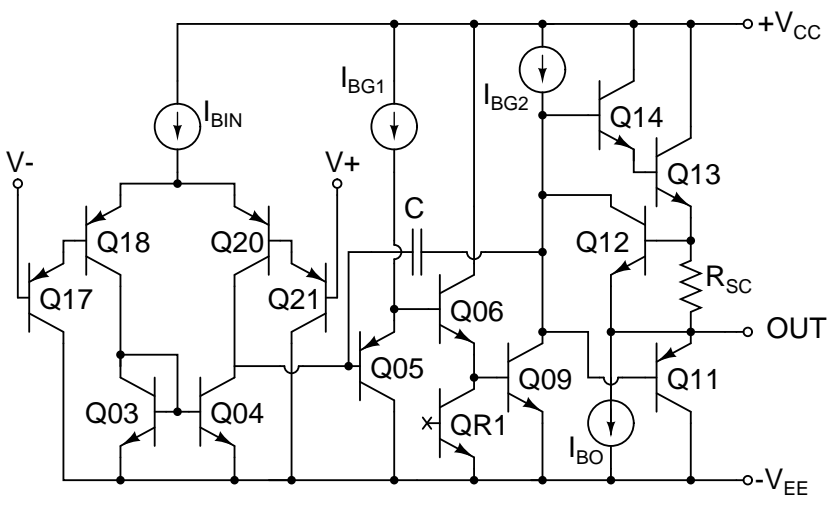

Fig. 3. Simplified LM124 diagram. The values or $I_{B I N}, I_{B G 1}, I_{B G 2}$ and $I_{B O}$ are $6,4,100 \& 50 \mu \mathrm{A}$. On the other hand, $R_{S C}=20 \Omega$. Finally, a resistor, connected to the base of Q09 and called R1 at the manufacturer's datasheet, turned out to be a open-base NPN transistor [20].

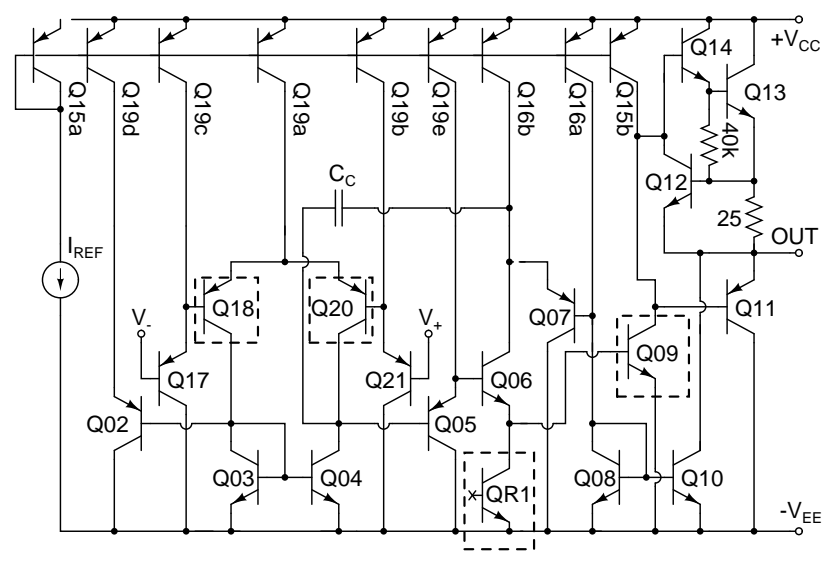

Fig. 4. Actual structure of the LM124 according to Savage et al. [20]. More information about the current source, $I_{R E F}$, can be found in the original paper. The transistors hit by the laser are highlighted.

\section{RESULTS}

Four points were chosen to investigate the effects of the resistive load on the shape on the laser induced transients. These points were the bases of Q09, Q18, Q20, and QR1, the first being a transistor of the gain stage, the other two transistors at the differential pair of the input stage and the last an open-base transistor working as a resistor in the gain stage. Fig. [3 shows the simplified structure provided by the manufacturer in the device datasheet. We have preferred not to follow the nomenclature used by the manufacturer but that used in most of the papers [18]. For instance, Q09 in Fig. [B is called Q12 at the datasheet by National Semiconductors [12]. The actual structure has been determined by several authors [7], [8], [10], [19], [20], although there are minor changes among the schematics provided by the different works. In particular, Fig. 团 shows the structure provided by Savage [20]. Finally, Fig. [ shows the exact placement of the transistors at which the laser was aimed.

Once the resistive load was set using the jumpers, a $z$ scan was performed with a step of $5 \mu \mathrm{m}$ penetrating in the device. At each depth value, the data after the laser impact were saved in the hard disk of the computer to be analyzed 


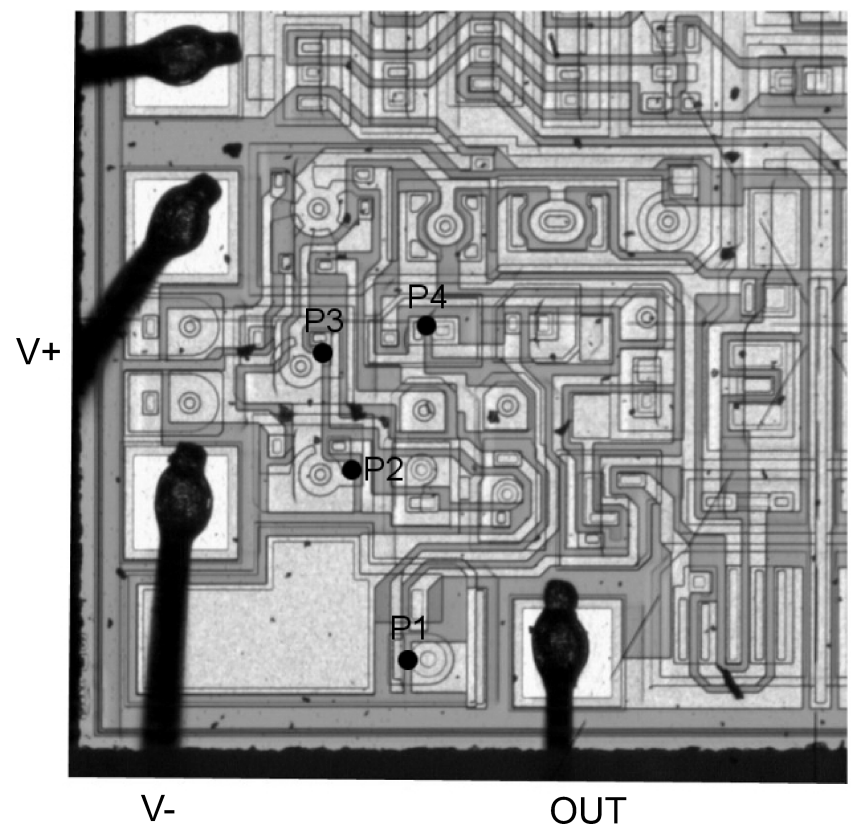

Fig. 5. Microphotograph of the LM124 lay-out. Spots where the laser was focused are marked with black dots. P1, P2, P3, and P4 are the bases of Q09, Q18, Q20, and QR1 [9].

by a specially developed SCILAB program in order to obtain typical parameters such as the peak voltage, full width-half maximum (FWHM), etc.

According to the results, there was not a significant difference among the output transients observed after hitting Q18 or Q20. Thus, similar transients to those depicted in the literature [9] were registered and the duration and size of the transients seemed to be independent of the load connected to the output. On the contrary, the behaviors of Q09 \& QR1 are by far much more interesting.

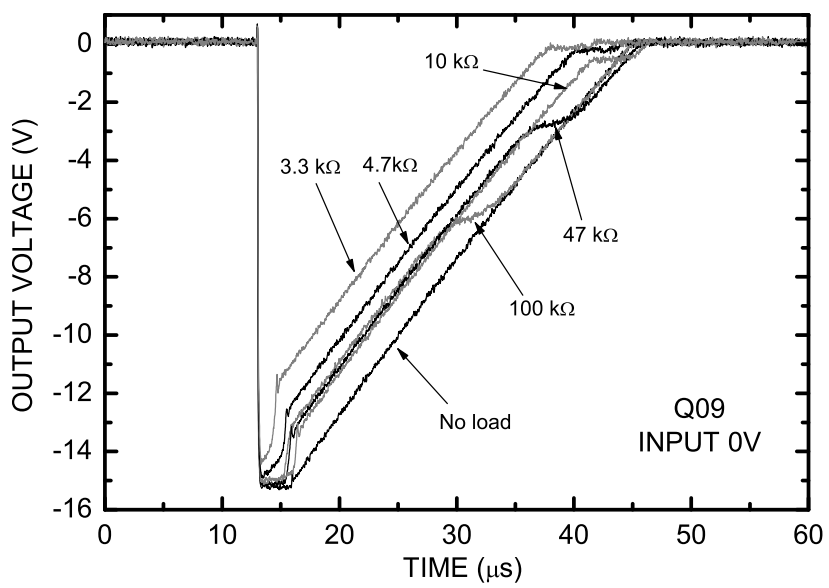

Fig. 6. Output transients observed after hitting Q09 at a depth of $30 \mu \mathrm{m}$. Every line is associated with a resistive load. The input was $0 \mathrm{~V}$.

\section{A. Transients at $Q 09$}

Transients related to this transistor are fast drops down to a value close to $-V_{E E}$ followed by a slower recovery

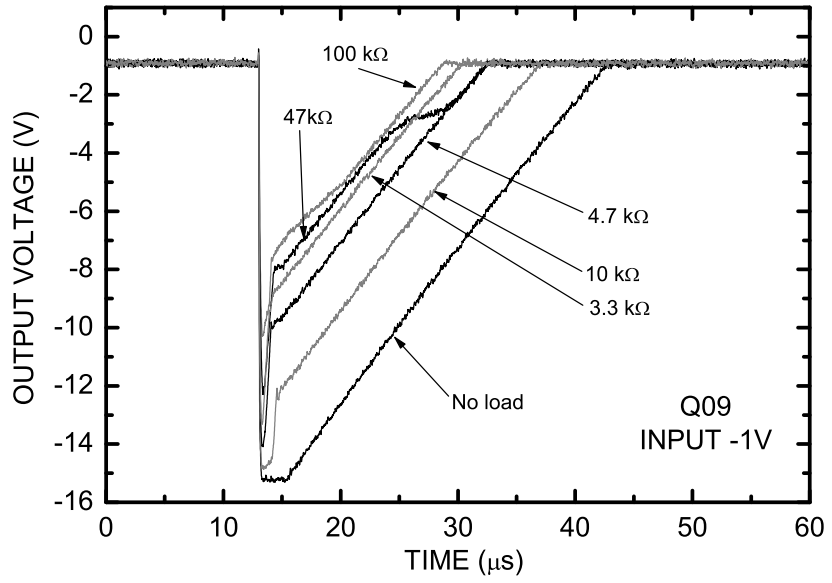

Fig. 7. Output transients registered after hitting the base of Q09 at a depth of $30 \mu \mathrm{m}$ with an input voltage of $-1 \mathrm{~V}$.

until reaching the DC output voltage, the speed of which is determined by the operational amplifier slew rate $(\sim 0.3$ $0.5 \mathrm{~V} / \mu \mathrm{s})$. Fig. 6 shows the modification of the single event transients as the load resistance decreases. In general, the effects of the resistive load are mainly two:

1) A fast recovery during the first steps of the transients before the signal changing according to the slew rate value.

2) A hump at the end of the transient, making the output value almost constant when $V_{O U T} / R_{L} \approx-60 \mu \mathrm{A}$.

Later, the input voltage switched to $V_{I N}=-1 \mathrm{~V}$, the results of this experiment being shown in Fig. $\square$. The main conclusions that we can derive from this figure are:

1) The transients of the unloaded amplifier seems to be independent of the input value with the only fact that transients with $V_{I N}=-1 \mathrm{~V}$ are a little shorter given that the DC output voltage is closer to the negative saturation voltage.

2) Loads of $47 \mathrm{k} \Omega \& 100 \mathrm{k} \Omega$ induce shorter and smaller transients than lower load resistor values.

3) Transients associated with loads of 3.3, $4.7 \& 10 \mathrm{k} \Omega$ do not have the characteristic hump observed in Fig. 6 .

In general, transients in unloaded amplifiers are bigger than those observed in loaded ones. Fig. \& shows that the points related to the transients approach to the $y$-axis as the load resistance decreases. Besides, very few transients reach the negative power supply value if the resistance value is low.

\section{B. Transients at $Q R 1$}

Those transients induced on the base of QR1 are usually short spikes that lead the output voltage up to the positive saturation voltage [8], [9]. They are followed by a swift decrease down to $0 \mathrm{~V}$, sometimes followed by a negative transient, smaller but longer. The reason of this transient is that the activation of QR1 takes the whole of the Q09 base current, momentarily cutting this transistor off so the output of the gain stage goes to a high positive value. These transients were recreated at the laser facility and the results of the 


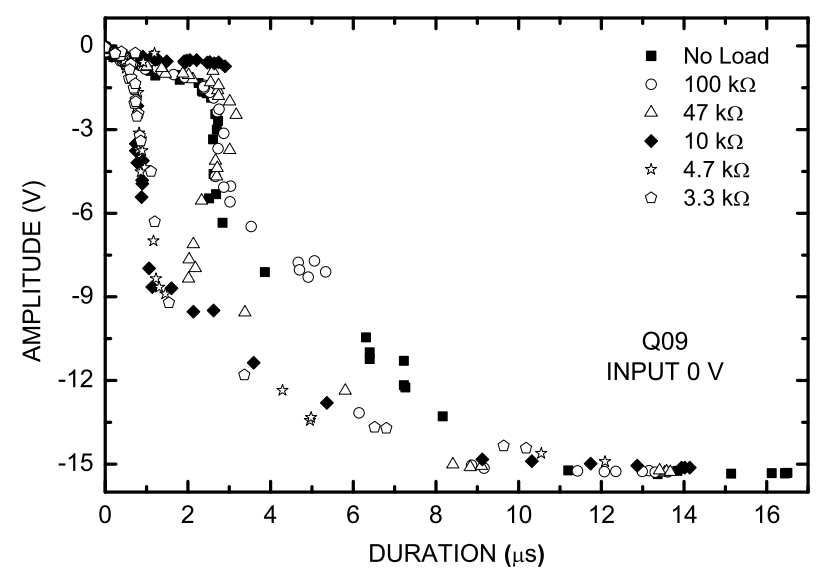

Fig. 8. Amplitude vs. FWHM of all the transients induced on the base of Q09. Once set the load resistor value and placed the laser over Q09, the laser performed a $z$-sweep to obtain a large set of transients with different shapes.
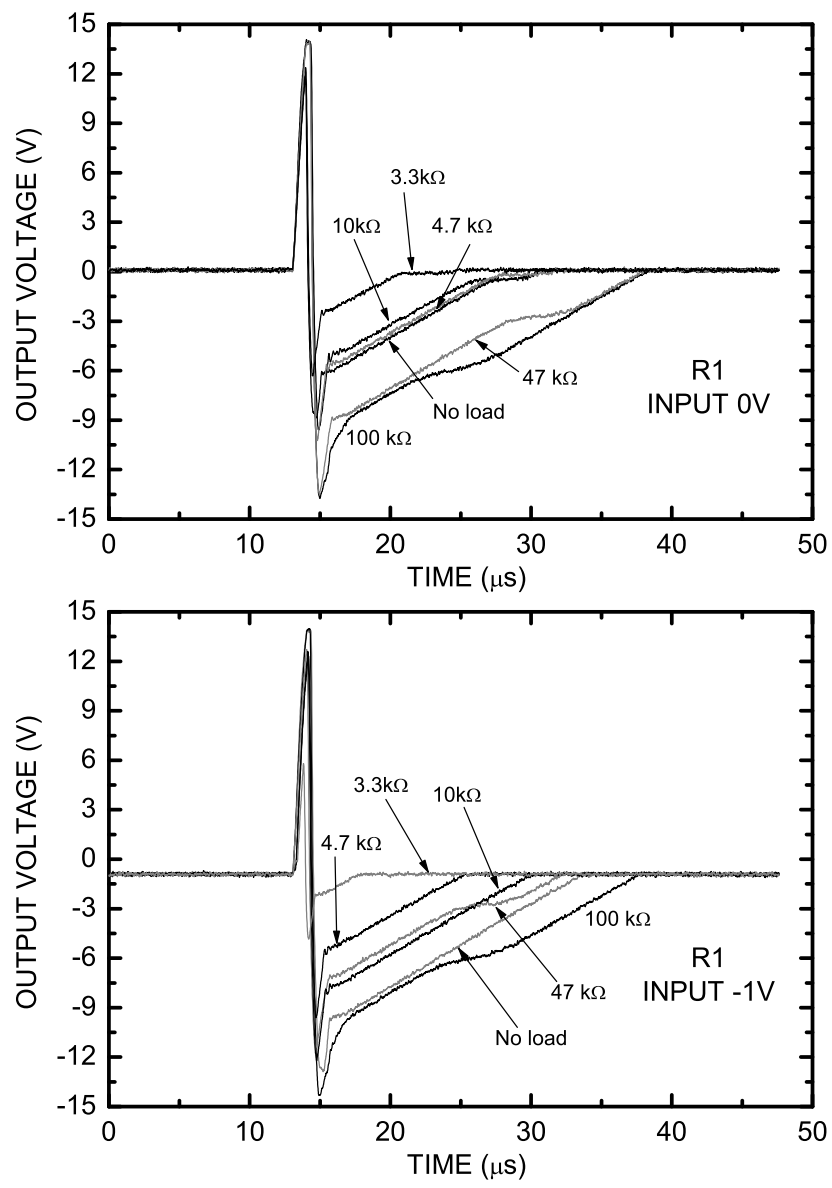

Fig. 9. Induced single event transients at a depth of $20 \mu \mathrm{m}$ below the base of QR1. The input voltage was $0 \mathrm{~V} \&-1 \mathrm{~V}$.

experiment are shown in Fig. Q. According to these results, it seems clear that the second stage of the transients, where the output voltage is negative, is attenuated as the load resistance decreases. However, in the case of using an input voltage of $-1 \mathrm{~V}$, the characteristics corresponding to loads of $100 \& 47$ $\mathrm{k} \Omega$ show humps resembling those depicted in the previous sections and making the transient longer than usual.
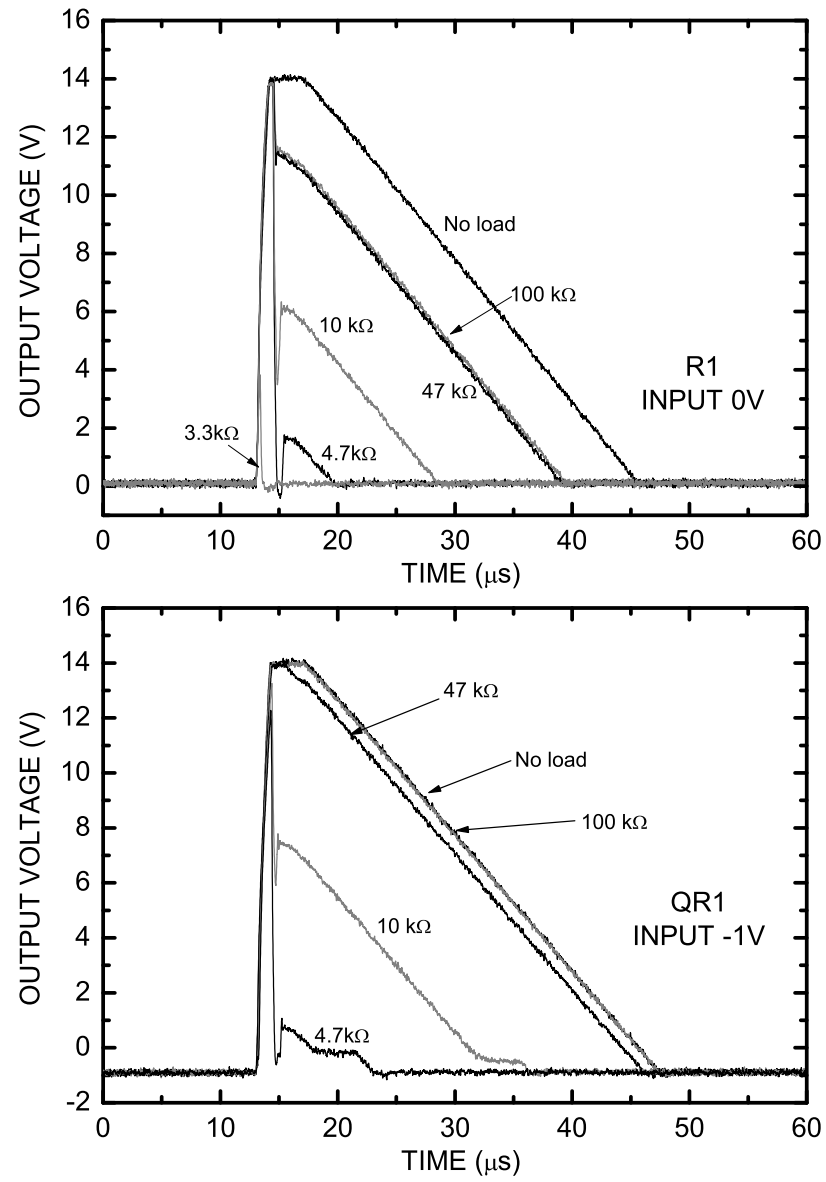

Fig. 10. Transients originated below QR1 at a depth of $30 \mu \mathrm{m}$ with an input of $0 \mathrm{~V} \&-1 \mathrm{~V}$ and different values of resistive loads.

Other interesting transients are those originated at a depth of $30 \mu \mathrm{m}$ (Fig. एव) , which are unusually large and strictly positive. Provided that these transients appears in a narrow interval of $z$-values, it is likely that these events are related to a charge generation near a buried layer.

If there is no load, the transient is just a quick increase followed by an interval at the positive saturation voltage finally, and ending with a slow decrease to a stable value accomplishing the slew rate limitation. Besides, the dependence of the transients on the input voltage is not significant. In fact, the transient associated with $V_{I N}=-1 \mathrm{~V}$ is longer since the DC value is a bit farther from the positive saturation voltage than the ground. However, as the load resistance decreases, the original transient seems to split up into two parts: The original spike associated with $\mathrm{QR} 1$, quite insensitive to the load, and a slower signal the size and duration of which is strongly affected.

The transients show some interesting facts: First of all, the line corresponding to a $3.3 \mathrm{k} \Omega$ resistor is just a short and small spike. In fact, most of the transients registered during the $z$ sweep with other loads vanished when this resistor value was used instead. Besides, lines related to the $4.7 \& 10 \mathrm{k} \Omega$ in Fig. 口] with $V_{I N}=-1 \mathrm{~V}$ also show a little hump prior to recover the original value. As in the case of $\mathrm{Q} 09$, this phenomenon occurred when $V_{O U T} / R_{L} \approx-60 \mu \mathrm{A}$. 


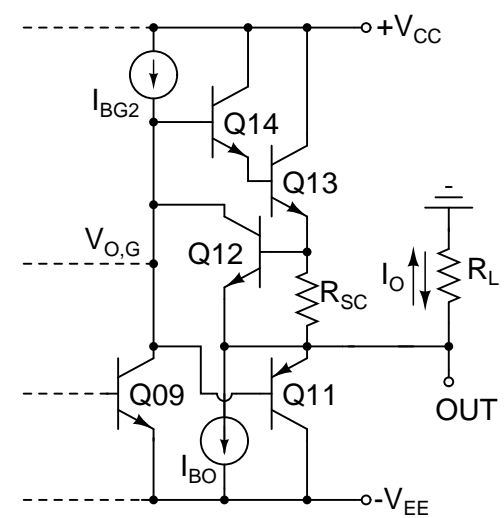

Fig. 11. Output stage of the LM124 with a load resistor.

\section{DISCUSSION}

Experimental data show that the load resistance affects the shape of the output transients. With the help of the analysis of the output stage and simulations, a theory to explain the distortions and attenuation of the single event transients, and based on the switch of some transistors to anomalous states, will be developed in the following sections.

\section{A. DC behavior of the output stage}

Unlike most of the typical general-purpose operational amplifiers that make use of a class AB output stage, the LM124 output stage is a combination of two emitter followers that cannot simultaneously work. The way that the stage operates depends on the value of the output current, $I_{O}$, (Fig. WI). In fact, if the output current is positive or, at least, negative but lower than $I_{B O}, \mathrm{Q} 13 \&$ Q14 must be in forward active zone to accomplish the Kirchoff's current law at the OUT node. Thus:

$$
V_{O U T} \approx V_{O, G}-2 V_{B E,(O N)}
$$

$V_{O, G}$ being the output of the gain stage (Q09 collector) and $V_{B E,(O N)}$ the voltage drop at a forward biased base-emitter junction (on the order or 0.6-0.7 V). A consequence of it is that $\mathrm{Q} 11$ is in cutoff state since $V_{E B, Q 11} \approx-1.2 \mathrm{~V}$ (The voltage drop along $R_{S C}$ is negligible). However, if the output current is negative and higher than $I_{B O}$, the only way to drain the excess of current towards $-V_{E E}$ is through Q11, a PNP transistor. This fact changes the operation point of the output stage since, in this situation, the mathematical relation between $V_{O, G}$ and $V_{O U T}$ becomes:

$$
V_{O U T} \approx V_{O, G}+V_{B E,(O N)} .
$$

In this case, Q13 \& Q14 switch to cutoff state since the voltage difference between the Q14 base and the Q13 emitter is on the order of $-0.6 \mathrm{~V}$. Finally, the purpose of Q12 is to protect the device from overcurrent. If something forces the operational amplifier to provide too much output current, a voltage difference appears between the base and the emitter of Q11 due to the presence of $R_{S C}$ in such a way that the Q12 collector grabs some current from the base of Q14 making the output current decrease. Besides, when the amplifiers does
TABLE I

RELATIVE TRANSISTOR AREA FOR SPICE SIMULATIONS. THE REST OF TRANSISTORS HAVE A RELATIVE AREA OF 1.0.

\begin{tabular}{llllll} 
Transistor & Area & Transistor & Area & Transistor & Area \\
\hline & & & & & \\
Q11 & 2.0 & Q16B & 1.3 & Q19C & 0.11 \\
Q15B & 3.0 & Q19A & 0.3 & Q19D & 0.12 \\
Q16A & 1.3 & Q19B & 0.11 & Q19E & 0.12 \\
\hline
\end{tabular}

not provide a lot of positive output current, the voltage drop through $R_{S C}$ is very low so the transistor remains in cutoff state. Finally, if Q11 is in forward-active zone instead of Q13 \& Q14, Q12 switches to reverse-active zone since the collector-base voltage is on the order of $0.6-0.7 \mathrm{~V}$.

\section{B. SPICE simulations}

SPICE simulations were performed in order to find out the reasons of the change of the SETs. The internal topology of the LM124 (Fig. (1) has been depicted in several papers [7], [8], [10], [19], [20] although scarce information is provided about the characteristic of the internal transistors. In fact, the typical procedure is separating the individual transistors by means of laser or ion beams and extract the SPICE parameters using a microprobe and specific instrumentation [18]. Unfortunately, this technology is not at the authors' disposal. However, given that the purpose of the simulations is to broadly understand the behavior of the device, we proceeded to do the following approaches:

1) All the NPN or PNP transistors were identical except area ratios.

2) Spice parameters were the typical values of a $5 \Omega-\mathrm{cm}, 17$ $\mu$-epi 44-V technology [21]. Junction capacitances were reduced in order to stabilize the amplifier.

3) Mirror transistor areas were trimmed so that the bias currents were those of Fig. [B].

The transients were simulated using an 1-ps rise \& fall time triangular current source between the involved nodes. The base resistance was removed of the transistor model and placed outside [22]. The by-pass capacitor was calculated by means of the slew rate value $(18 \mathrm{pF})$ [23], in agreement with the value suggested by other authors [20].

Even after all these simplifications, the results are meaningful. Fig. [D shows the simulation of a SET on the bases of Q09 \& QR1, which are similar to the experimental results (Fig. (6)). In general, the simulations recreate most of the experimental results except those related to parasitic elements, not included in the SPICE netlist. This way, most of the typical QR1 SETs were emulated except those large transients depicted in Fig. [1. Probably, the reason of this failure is that a unique current source cannot modelate a multi-junction charge collection process [24].

\section{Transients at $Q 09$}

Usually, the distortion of the transients related to the change of the load resistance, either pure load or resistive network, are explained supposing a coupling effect. However, a careful study of the behavior of the transistors during the transients 

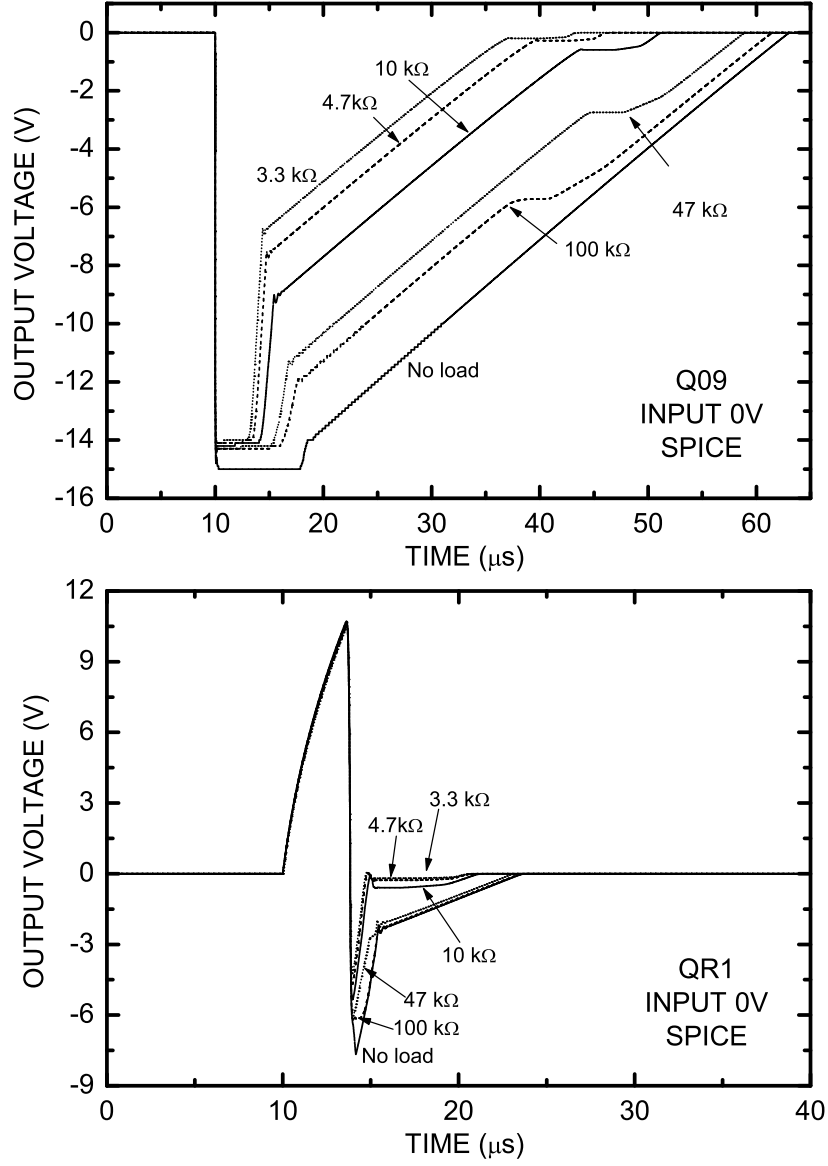

Fig. 12. Simulated SETs on the base of Q09 \& QR1 with grounded input. Injected charge was $10 \mathrm{pC}$ in the case of Q09 and $2 \mathrm{pC}$ in QR1. In both simulations, the transistor base resistance is $1 \mathrm{k} \Omega$. The simulations were performed using two engines to verify the results, the GNU NGSpice Rework19, and the LTSpice by Linear Instruments.

allows understanding that the actual reason is that, temporarily, some transistors give up working in the correct mode making the whole device not operating in the typical zone. E. g., transistors supposed to be in forward active zone switch to saturation or alike.

Transients at Q09 are quite simple and were successfully emulated by the SPICE simulations so its study will help to understand the internal processes that yielded the SET distortion. In this transients, three stages are clearly distinguished: Saturated output, slew-rate controlled evolution and humps.

1) Saturated output voltage and slew-rate controlled evolution: Few microseconds after the hit, the output and the inverting input of the operational amplifier plunge down to the negative saturation voltage. Immediately, the operational amplifiers undergoes the following changes: First of all, Q09 goes to saturation state and most of the charge stored at the bypass capacitor is removed. Simultaneously, the sudden fall of the inverting input voltage ends up forcing Q04 \& Q05 to enter in a anomalous situation. Due to the previous accumulation of charge in reverse biased PN junctions and the drop of the Q04 base voltage to a value near $-V_{E E}$, the Q04 collector voltage temporarily falls below $-V_{E E}$ so this charge starts to be transferred to the now reverse biased Q05 base-emitter junction (Fig. [3). In the output stage, if there is a small load resistance, Q11 starts to absorb current and Q12 quits the cutoff state (Section $\mathbb{R - A}$ ).

In case of not existing a resistive load, this temporary situation ends when the $100 \mu \mathrm{A}$ current source manages to provide enough charge to allow the switching of Q09 from saturation to forward-active zone. Usually, this is a relative slow process that lets Q04 \& Q05 come back to stable states allowing the input differential pair to work properly. Following this, the charge removed by the hit on the capacitor is stored again by the Q03 \& Q04 current mirror so the change rate is constant and equal to the slew rate value. Indeed, the op amp response is similar to that of an input voltage switch from large negative to positive values.

However, this background is not valid in case of existing a load. Simulations show that a fraction of the output current coming from the resistive load does not follow the typical path (the Q11 emitter) but is absorbed by Q12, in reverse active zone. This current eventually arrives at the Q09 collector making faster the change of Q09 from saturation to forward active zone. Therefore, the gain stage quickly recovers its correct operation point. However, neither Q04 nor Q05 have managed to reach the stable state so, for a few microseconds, they absorb much more current than expected due to very low voltage value of the Q04 collector. This current is taken from the by-pass capacitor to bias again the Q04 \& Q05 PN junctions. Thus, the recovery of the output voltage is faster than that of the typical, controlled by the input stage current source. Eventually, both Q04 \& Q05 return to stable state and the output voltage begins to change according to the slew rate value. In any case, the smaller the load resistance, the faster the recovery during this stage.

2) Presence of humps: Now, let us depict the behavior of the transient during its last stage. When the output current is on the order of $-50 \mu \mathrm{A}$, there is not enough current to be shared by $I_{B O}, \mathrm{Q} 11 \& \mathrm{Q} 12$ (Fig. $\left.\mathrm{W}\right)$. In this situation, Q11 \& Q12 switch from reverse-active to cutoff state whereas the Q13 \& Q14 Darlington pair come back to forward active zone. During this interval, the output stage stops working so the output voltage keeps constant until leaving this blind zone. This is the explanation of the strange slowing of the output transient recovery during the final stage of the transient, as it can be clearly observed between $35-45 \mu$ s in the $47-\mathrm{k} \Omega$ characteristic of Fig. 6 as well in the simulations. Given this resistance value and that the value of the output voltage was about $-3 \mathrm{~V}$, the output current was $-3 / 47=-64 \mu \mathrm{A}$, a value that accurately fits the forecast output current to make Q12 switch off. This hump was not observed in the unloaded amplifier since, in this case, the Q13 \& Q14 Darlington pair never switched to cutoff state. On the other hand, the distortion was also observed in amplifiers with small load resistances but occurring at lower output voltage values. Actually, humps are present in other characteristics in Fig. 6 although they are quite imperceptible.

These humps were observed by Buchner et al. after studying the effects of total ionizing dose on the shape of the LM124 transients [17]. These authors reported that the humps or plateaus appeared at the Q09 transients if the initially positive output voltage crosses a critical threshold of $-0.6 \mathrm{~V}$. They 
concluded that the reason of this behavior was a change to large-signal regime, fact that agrees with the discussion developped in this paper.

3) Influence of the input voltage: Finally, if $V_{I N}=-1 \mathrm{~V}$, the DC output current with $10,4.7 \& 3.3 \mathrm{k} \Omega$ is $-100,-213$ $\&-516 \mu \mathrm{A}$. In other words, the Q11 transistor always works so no swap is needed between Q13 \& Q14 and Q11 in such a way that humps lack. This is the reason of the absence of humps in the experimental data related to these loads (Fig. Ш). However, simulations fail to predict the little size of the transients associated with the intermediate resistance values. In fact, according to the predictions these transients should be placed between the not-loaded and the low resistor load graphs. The reason of this behavior is unknown.

The influence of the input voltage on the transients agrees with the results reported by Buchner et al. [17].

\section{Transients at $Q 18 \& Q 20$}

As it was previously said, transients induced at the transistors Q18 and Q20 were not affected by the presence of a load. However, this fact is not surprising since the gain stage is a buffer block that insulates the output stage, strongly influenced by the load resistance, and the input stage where the transients were induced. Besides, these transients were smaller than those observed in the output stage so the effects previously illustrated cannot take place.

\section{E. Transients at $Q R I$}

In the typical single event transients related to this spot, the trend is that small resistance values minimize the size of the transients, in particular during the second period where the output voltage reaches large negative values. Depending on the resistance value, the appearance of humps can increase the duration of some of the single event transients. Just like the Q09 transients, these humps vanish with appropriated values of input voltage due to the fact that there is no switching among the output stage transistors. This fact explains that 4.7$\mathrm{k} \Omega$ load SETs are longer if the input voltage is $0 \mathrm{~V}$ than if it is $-1 \mathrm{~V}$. Finally, no explication was found to understand the strange behavior of the unloaded operational amplifier. Indeed, simulations predict that these transients should be longer than in the case of loaded operational amplifiers. However, the experiment was repeated and this behavior verified.

Regarding the anomalous set of single events observed in deeper spots, simulation could not help to understand them given that these transients were originated at some parasitic element not included in the SPICE model. However, it must be highlighted the fact that the QR1 transients also show humps when the DC output voltage is negative and the two parts of the output stage switches. Thus, comparing the characteristics of $4.7 \mathrm{k} \Omega$ in Fig. 四, the duration of the transient is affected by the output voltage value. A grounded input makes the transient last for only $7 \mu \mathrm{s}$ whereas an input voltage of $-1 \mathrm{~V}$ leads to a transient of about $10 \mu \mathrm{s}$.

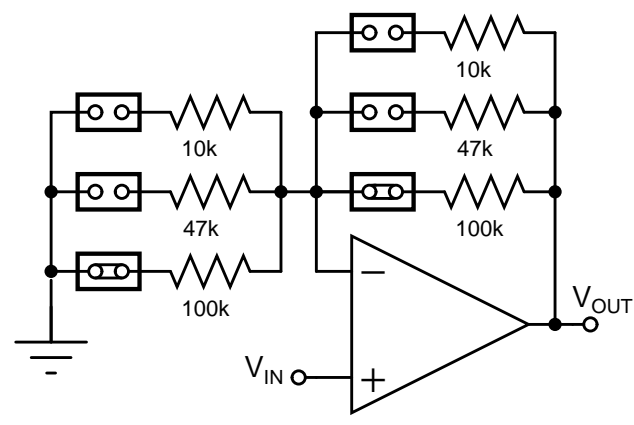

Fig. 13. Electric configuration to investigate the LM124 SETs with constant gain equal to 2 and different resistor values. The use of jumpers allows selecting the desired resistor values.

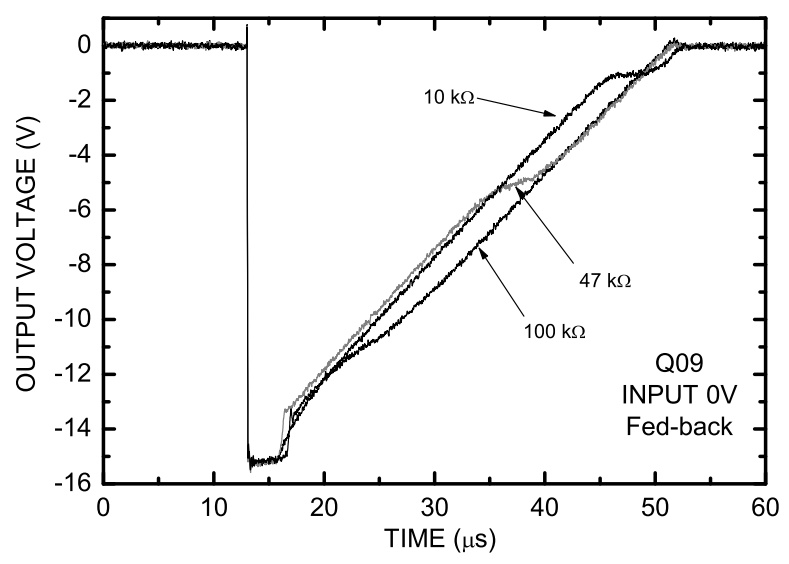

Fig. 14. Single event transients induced by laser hit at a depth of $25 \mu \mathrm{m}$ below Q09. The amplifier was fedback with a gain of 2 using the resistor value related to each graph.

\section{F. Effects on fed-back operational amplifiers}

Experimental results have shown that a load resistance can affect the characteristics of the single event transients originated at the gain stage. The tested device was configured as Fig. \ shows, with a load connecting the device output and ground. However, operational amplifiers can have resistive loads in some situations not as clear as that shown in this figure. Let us suppose that the amplifier is in a typical noninverting configuration, with two resistors $R_{1} \& R_{2}$ in such a way that the gain is $1+R_{2} / R_{1}$. In this framework, a resistor equivalent to $R_{1}+R_{2}$ loads the amplifier.

Laser tests were done using a feedback network with $R_{1}=$ $R_{2}=R, R$ being $100,47 \& 10 \mathrm{k} \Omega$ in order to investigate if the single event transients are affected by the values of the resistors of the electrical network (Fig. [13). The conclusion of these tests were that, from an end-user's point of view, there was not an appreciable difference between the response of the fed-back amplifier with different values of $R$. The reason is that, in the LM124, load effects become significant when the load values were below 5-10 $\mathrm{k} \Omega$. In other words, changes in the single event transients would appear if $R$ were on the order of 2-5 $\mathrm{k} \Omega$. However, this is not realistic since it is uncommon to use such low resistance values to feedback an operational amplifier due to the penalty on the power consumption of the system. 
Nevertheless, single event transients are doubtless distorted by the load. Fig. [4] illustrates the evolution of the single event transients of a fed-back operational amplifier as the resistor value decreases. According to the graph, the transients show similar characteristics observed on the loaded amplifiers: A fast increase after quitting the negative saturation voltage before the recovery accomplishing the slew rate requirement, and the presence of humps when the output value is on the order of $-50 \mu \mathrm{A} \cdot\left(R_{1}+R_{2}\right)$. Given that both resistors are equal, the previous expression becomes $V_{H U M P} \approx-0.1 \cdot R$, $R$ being expressed in $\mathrm{k} \Omega$. Paradoxically, the presence of the hump makes the transient with $10 \mathrm{k} \Omega$ a little longer than the others, even though its value of FWHM is clearly lower.

Finally, it is interesting to cite previous works where the presence of humps related to the load resistors is perceived. E. g., Sternberg et al. found that the network resistor values clearly modify the size and shape of the simulated transients [5]. They forecast a decrease of the size and duration of single event transients in an inverting network if small resistance values are used. The explanation given by the authors was that there was a coupling between the resistance values, gain, and bandwidth. Nevertheless, the load effect theory developed in this paper could explain this behavior as well. For instance, single event transients on Q09 reported in that paper, with network resistors of $10 \& 1 \mathrm{k} \Omega$, show a strange slowing when the output voltage is about $-0.5 \mathrm{~V}$. That means that the output current is $-45 \mu \mathrm{A}$, in agreement with the results shown here.

\section{CONCLUSION}

Some of the typical transients observed at the output of the LM124 operational amplifiers after a pulsed laser impact are modified by the presence of a resistive load at the output. The affected output transients seems to be shorter and smaller than those observed without any resistive load. Besides, the distortion depends on the DC output voltage. This behavior is related to two facts: First of all, the current associated with the load resistor helps the internal transistors to recover the original state, and also the output stage cannot instantaneously change its operation point to bias the load resistor.

Finally, tests on fed-back amplifiers were performed to find out if the resistor values affected the shape of the output transients. As expected, load effects related to the feed-back network are evident so this phenomenon must be taken into account along with the shift of the operational amplifier poles and zeros to explain the distortion of the LM124 SETs.

\section{ACKNOWLEDGMENT}

The authors would like to thank Dr. J. M. Miranda Pantoja, at the Departamento de Física Aplicada III of the Universidad Complutense de Madrid, for his support during the development of the data acquisition system.

\section{REFERENCES}

[1] C. Poivey, et al., "Development of a test methodology for single-event transients (SETs) in linear devices," IEEE Transactions on Nuclear Science, vol. 48, no. 6, pp. 2180-2186, Dec. 2001.
[2] Y. Boulghassoul, et al., "Circuit modeling of the LM124 operational amplifier for analog single-event transient analysis," IEEE Transactions on Nuclear Science, vol. 49, no. 6, pp. 3090-3096, Dec. 2002.

[3] D. McMorrow, et al., "Subbandgap laser-induced single event effects: carrier generation via two-photon absorption," IEEE Transactions on Nuclear Science, vol. 49, no. 6, pp. 3002-3008, Dec. 2002.

[4] R. L. Pease, et al., "Comparison of SETs in bipolar linear circuits generated with an ion microbeam, laser light, and circuit simulation," IEEE Transactions on Nuclear Science, vol. 49, no. 6, pp. 3163-3170, Dec. 2002.

[5] A. L. Sternberg, et al., "Effect of amplifier parameters on single-event transients in an inverting operational amplifier," IEEE Transactions on Nuclear Science, vol. 49, no. 3, pp. 1496-1501, Jun. 2002.

[6] Y. Boulghassoul,et al., "Investigation of millisecond-long analog single-event transients in the LM6144 op amp," IEEE Transactions on Nuclear Science, vol. 51, no. 6, pp. 3529-3536, Dec. 2004

[7] S. Buchner, J. J. Howard, C. Poivey, D. McMorrow, and R. Pease, "Pulsed-laser testing methodology for single event transients in linear devices," IEEE Transactions on Nuclear Science, vol. 51, no. 6, pp. 3716-3722, Dec. 2004.

[8] S. Buchner, et al., "Comparison of single-event transients induced in an operational amplifier (LM124) by pulsed laser light and a broad beam of heavy ions," IEEE Transactions on Nuclear Science, vol. 51, no. 5, pp. 2776- 2781, Oct. 2004.

[9] D. McMorrow, et al., "Demonstration of single-event effects induced by through-wafer two-photon absorption," IEEE Transactions on Nuclear Science, vol. 51, no. 6, pp. 3553-3557, Dec. 2004.

[10] P. C. Adell, et al., "Single event-induced instability in linear voltage regulators," IEEE Transactions on Nuclear Science, vol. 53, no. 6, pp. 3506-3511, Dec. 2006.

[11] C. Weulersse, et al., "Probing SET sensitive volumes in linear devices using focused laser beam at different wavelengths," IEEE Transactions on Nuclear Science, vol. 55, no. 4, pp. 2007-2012, Aug. 2008.

[12] "National semiconductors's website." This document is avalaible on-line at http://www.national.com/mpf/LM/LM124.html.

[13] A. H. Johnston, G. M. Swift, T. F. Miyahira, and L. D. Edmonds, "A model for single-event transients in comparators," IEEE Transactions on Nuclear Science, vol. 47, no. 6, pp. 2624-2633, Dec. 2000.

[14] S. D. LaLumondiere, R. Koga, P. Yu, M. C. Maher, and S. C. Moss, "Laser-induced and heavy ion-induced single-event transient (SET) sensitivity measurements on 139-type comparators," IEEE Transactions on Nuclear Science, vol. 49, no. 6, pp. 3121- 3128, Dec. 2002.

[15] C. Poivey, S. Buchner, J. Howard, K. A. Label, and R. Pease, "Testing and hardness assurance guidelines for single event transients (SET) in linear devices," Rep. NASA GSFC, 2005. [Online]. Available: http://radhome.gsfc.nasa.gov/radhome/papers/SET_Linear05.pdt

[16] Y. Boulghassoul, et al., "System-level design hardening based on worstcase ASET simulations," IEEE Transactions on Nuclear Science, vol. 51, no. 5, pp. 2787-2793, Oct. 2004.

[17] S. Buchner, D. McMorrow, N. Roche, L. Dusseau, and R. Pease, "The effects of low dose-rate ionizing radiation on the shapes of transients in the LM124 operational amplifier," IEEE Transactions on Nuclear Science, vol. 55, no. 6, pp. 3314-3320, Dec. 2008.

[18] R. Pease, "Modeling single event transients in bipolar linear circuits," IEEE Transactions on Nuclear Science, vol. 55, no. 4, pp. 1879-1890, Aug. 2008.

[19] L. Bonora and J. David, "An attempt to define conservative conditions for total dose evaluation of bipolar ics," IEEE Transactions on Nuclear Science, vol. 44, no. 6, pp. 1974-1980, Dec. 1997.

[20] M. Savage, et al., "Variations in SET pulse shapes in the LM124A and LM111," 2002 IEEE Radiation Effects Data Workshop, pp. 75-81, 2002.

[21] P. R. Gray and R. G. Meyer, Analysis and Design of Analog Integrated Circuits, 3rd ed. USA: John Wiley \& Sons Inc., 1993, ch. 2, pp. 123-147.

[22] A. L. Sternberg, et al., "The role of parasitic elements in the single-event transient response of linear circuits," IEEE Transactions on Nuclear Science, vol. 49, no. 6, pp. 3115-3120, Dec. 2002.

[23] F. J. Franco, Y. Zong, and J. Agapito, "New details about the frequency behavior of irradiated bipolar operational amplifiers," IEEE Transactions on Nuclear Science, vol. 53, no. 4, pp. 1931-1938, Aug. 2006.

[24] D. McMorrow, et al., "Three-dimensional mapping of single-event effects using two photon absorption," IEEE Transactions on Nuclear Science, vol. 50, no. 6, pp. 2199-2207, Dec. 2003. 